\title{
Factores ecológicos asociados con la presencia de larvas de Aedes aegypti en zonas de alta infestación del municipio Playa, Ciudad de La Habana, Cuba
}

\author{
Juan Andrés Bisset Lazcano, ${ }^{1}$ María del Carmen Marquetti, ${ }^{1}$ Reina Portillo, ${ }^{2}$ \\ María Magdalena Rodríguez, ${ }^{1}$ Silvia Suárez ${ }^{1}$ y Maureen Leyva ${ }^{1}$
}

Forma de citar

Bisset Lazcano JA, Marquetti MC, Portillo R, Rodríguez MM, Suárez S, Leyva M. Factores ecológicos asociados con la presencia de larvas de Aedes aegypti en zonas de alta infestación de Ciudad de La Habana, Cuba. Rev Panam Salud Publica. 2006;19(6):379-84.

RESUMEN Objetivos. Identificar los factores ecológicos locales que pudieron incidir en la mayor infestación vectorial con Aedes aegypti observada en cuatro áreas de salud urbanas de Ciudad de La Habana, Cuba, así como los tipos de depósitos con mayor número de larvas.

Métodos. Estudio descriptivo en cuatro áreas de salud (Docente, 28 de Enero, 26 de Julio y 1. ${ }^{\circ}$ de Enero) del municipio Playa, Ciudad de La Habana, Cuba, seleccionadas por la presencia reiterada del vector del dengue en el año 2003. Entre abril de 2004 y marzo de 2005 se inspeccionaron todos los locales de las manzanas seleccionadas. Se caracterizaron los depósitos existentes con agua (tipo, cantidad y ubicación) y los factores ecológicos observados (existencia de patio y de sombra, forma en que estaban tapados los tanques, presencia de materia orgánica en ellos e higiene de la vivienda). Se contaron las larvas y pupas de mosquitos de cada depósito mediante filtración. La identificación taxonómica de las pupas se realizó con microscopio estereoscópico.

Resultados. Los depósitos en que se encontraron larvas y pupas de A. aegypti con mayor frecuencia en todas las áreas fueron los tanques bajos y los pequeños depósitos artificiales. En tres de las áreas estudiadas la mayor cantidad de pupas se encontró en los tanques bajos $(88,6 \%, 100 \%$ y $56,6 \%)$, de los cuales $90,9 \%$ se encontraban destapados o tapados solo parcialmente, mientras que en el área restante los pequeños depósitos artificiales fueron los más frecuentes $(85,7 \%)$. Se encontró correlación entre el número de depósitos infestados en las viviendas con la presencia de patio $\left(\chi^{2}=29,59 ; \mathrm{P}=0,0001\right)$, de sombra parcial $\left(\chi^{2}=4,108 ; \mathrm{P}=\right.$ $0,0001)$, de vegetación $\left(\chi^{2}=43,59 ; \mathrm{P}=0,0001\right)$ y de árboles $\left(\chi^{2}=101,459 ; \mathrm{P}=0,0001\right)$, así como con la mala higiene $\left(\chi^{2}=53,76 ; \mathrm{P}=0,0001\right)$.

Conclusión. Los depósitos artificiales, especialmente los tanques bajos y los recipientes pequeños, son las criaderos más frecuentes de A. aegypti, por lo que constituyen un factor de riesgo de infestación con ese vector. Los tanques destapados con materia orgánica y situados a la sombra y en el exterior fueron los más peligrosos en ese sentido.

Palabras clave Aedes, control de mosquitos, vigilancia epidemiológica, Cuba.

Departamento de Control de Vectores, Instituto de Medicina Tropical Pedro Kourí, Centro Colaborador OPS/OMS para el Estudio del Dengue y su Vector, Ciudad de La Habana, Cuba. La correspondencia debe enviarse a Juan A. Bisset a: Depar- tamento de Control de Vectores, Instituto de Medicina Tropical Pedro Kourí, Autopista Novia del Mediodía km 61/2, Apartado postal Marianao 13, Ciudad de La Habana, Cuba. Correo electrónico: bisset@ipk.sld.cu
2 Departamento de Control de Vectores, Centro Provincial de Higiene y Epidemiología, Matanzas, Cuba. 
El mosquito Aedes aegypti es el vector más importante en la transmisión de la fiebre amarilla, el dengue y el dengue hemorrágico en la Región de las Américas. La primera epidemia de dengue ocurrió en Cuba en 1977 (serotipo 1) y provocó 4 millones de casos; en la segunda, en 1981 (serotipo 2), enfermaron 344203 personas, de ellas 10312 de gravedad, y se registraron 158 muertes. Esto llevó a establecer la Campaña Nacional de Erradicación de Aedes aegypti en junio de 1981. A partir de ese momento se produjo una brusca reducción de la densidad de ese vector gracias al empleo de insecticidas -tanto larvicidas como adulticidas-, al saneamiento ambiental, a la adopción de medidas jurídicas y a la participación activa de la comunidad (1).

A pesar de que este programa ha logrado controlar el vector en la mayor parte del país, aún quedan áreas donde el nivel de infestación es elevado. Estas áreas constituyen importantes factores de riesgo para la aparición de brotes de dengue, como el que afectó a las provincias de Santiago de Cuba y Ciudad de La Habana en 1997 y 2002, respectivamente (2). En esa ocasión no se implementaron con antelación las medidas de emergencia previstas, ya que la transmisión ocurrió sin que el índice domiciliario (porcentaje de casas con larvas con relación al total de casas inspeccionadas) y el índice de Breteau (porcentaje de depósitos con larvas con relación al total de casas inspeccionadas), calculados como parte del proceso habitual de vigilancia larval, sobrepasaran el valor umbral establecido de 5 .

En la actualidad se ejecuta un proyecto multicéntrico internacional auspiciado por la Organización Mundial de la Salud que evalúa índices nuevos y más eficaces, entre ellos uno basado en la detección de pupas del mosquito, y su correlación con los índices larvales y con la población adulta de $A$. aegypti en zonas de alto riesgo de transmisión. El objetivo es encontrar un índice que refleje mejor la presencia de mosquitos adultos en las áreas en riesgo de transmisión (3-6).

Otra medida para reducir la aparición de brotes epidémicos de dengue y para mejorar la eficacia en función del costo de las campañas contra Aedes consiste en identificar los factores ambientales locales que facilitan la reinfestación con el vector y mejorar la vigilancia y el muestreo de las formas tempranas del vector en esas áreas.

El objetivo de este trabajo fue identificar los factores ecológicos locales que pudieron incidir en la mayor infestación vectorial con $A$. aegypti observada en cuatro áreas de salud urbanas de Ciudad de La Habana, Cuba, así como los tipos de depósitos con mayor cantidad de larvas.

\section{MATERIALES Y MÉTODOS}

Se realizó un estudio descriptivo en cuatro de las nueve áreas de salud en que está distribuida la población del municipio Playa, al noroeste de la Ciudad de La Habana, Cuba. Las áreas estudiadas correspondieron a los policlínicos Docente, 28 de Enero, 26 de Julio y $1 .^{\circ}$ de Enero, y se seleccionaron por la presencia reiterada del vector del dengue entre enero y marzo de 2004, según la información ofrecida por el Centro Municipal de Higiene y Epidemiología de Playa. Dentro de cada área se seleccionaron las tres manzanas de mayor incidencia focal. El municipio Playa cuenta con una población de 197536 habitantes distribuida en una superficie de $3617 \mathrm{~km}^{2}$ (5 461 habitantes por $\mathrm{km}^{2}$ ).

En el área de salud 28 de Enero se inspeccionaron 240 viviendas donde vivían 780 personas (3,25 personas por vivienda); de las viviendas inspeccionadas, $23(9,6 \%)$ eran apartamentos y 217 $(90,4 \%)$ eran casas. Dos de las tres manzanas muestreadas en esta área de salud se encontraban en uno de los barrios insalubres del municipio. De las 408 viviendas inspeccionadas en el área Docente, $232(56,9 \%)$ eran casas y 176 eran apartamentos $(43,1 \%)$; en ellas vivían 924 personas (2,26 personas por vivienda). En el área $10^{\circ}$ de Enero se inspeccionaron 265 viviendas con 628 personas (2,37 personas por casa); 131 $(49,4 \%)$ de las viviendas eran apartamentos y $134(50,6 \%)$ eran casas. En el área del policlínico 26 de Julio se inspeccionaron 327 viviendas; de ellas, 112 eran apartamentos $(34,3 \%)$ y 215 eran casas $(65,7 \%)$. En ellas vivían 728 habitantes (2,23 personas por vivienda).

\section{Muestreo entomológico}

Entre abril de 2004 y marzo de 2005 se inspeccionaron todos los locales de las manzanas seleccionadas. Los resultados del muestreo se registraron en dos modelos confeccionados para la investigación: uno para los datos relativos a los locales inspeccionados (dirección de la vivienda, tipo, cantidad y ubicación de los recipientes con agua en cada vivienda) y otro para recoger los factores ecológicos (variables) que incidían en los recipientes en los que se encontraron larvas (existencia de patio y de sombra, forma en que estaban tapados los tanques, presencia de materia orgánica en ellos y de vegetación y árboles en su entorno e higiene de la vivienda). La cantidad de materia orgánica en los depósitos y la higiene de las viviendas se evaluaron mediante la apreciación del inspector según una escala cualitativa de tres puntos. Se consideró que había árboles si cerca del depósito se encontraba al menos un árbol de más de $4 \mathrm{~m}$ de altura.

Se contaron las larvas y pupas de mosquitos de cada depósito infestado mediante filtración. Las larvas se conservaron en alcohol al $70 \%$ y se enviaron al Centro Municipal de Higiene y Epidemiología de Playa para su identificación taxonómica. Las pupas se enviaron en viales plásticos con agua al Instituto de Medicina Tropical Pedro Kourí para su identificación. Todas las muestras se rotularon con etiquetas que indicaban el tipo de depósito, el lugar y la fecha de la colecta. La identificación taxonómica de las pupas se realizó con un microscopio estereoscópico y se prestó especial atención a las trompas respiratorias y las paletas anales, ya que estas estructuras se mantienen generalmente íntegras a pesar de la manipulación y el traslado.

\section{Clasificación de los depósitos}

Según la clasificación de Armada y colaboradores (7) se buscaron ocho 
tipos de depósitos: tanques bajos, floreros, vasos espirituales, cisternas, neumáticos, bebederos de animales, pequeños depósitos artificiales (latas, pomos, cubos, etc.) y otros depósitos (charcos, alcantarillas, zanjas y fosas). No se tuvieron en cuenta los tanques elevados por lo difícil de su inspección.

Para el análisis comparativo de los factores ecológicos de las áreas de salud estudiadas se utilizó la prueba de la ji al cuadrado. Se empleó un nivel de significación de 0,05.

\section{RESULTADOS}

En total se inspeccionaron 1240 viviendas en las que vivían 3060 personas $(1,55 \%$ de la población total del municipio). Se encontraron 7367 depósitos de agua y en 35 (0,48\%) de ellos se detectaron larvas o pupas del mosquito $A$. aegypti. Debido a que se seleccionaron las áreas de salud con mayor infestación, estos resultados no reflejan la situación epidemiológica del municipio Playa en su conjunto, sino de su sector más afectado. A continuación se exponen los resultados encontrados en cada área de salud.

\section{Área de salud Docente}

En esta área se revisaron en total 1725 depósitos (4,22 depósitos por casa) y solo en tres locales no se almacenaba agua en ningún tipo de recipiente. Los tipos de depósito más frecuentes fueron los tanques bajos (638) y los artificiales (634), mientras que los menos frecuentes fueron los neumáti$\cos (22)$.

Se encontraron 8 recipientes infestados (cuadro 1), todos con pupas. La mayor cantidad de ellas $(56,7 \%)$ se encontró en los tanques bajos, seguidos de los neumáticos (20\%), los pequeños depósitos artificiales $(16,7 \%)$ y los bebederos (6,7\%). El 87,5\% de los depósitos encontrados estaban situados en los patios de las viviendas. Todos contenían abundante materia orgánica y estaban parcial $(62,5 \%)$ o totalmente $(37,5 \%)$ a la sombra. Se observó la pre-

CUADRO 1. Características y ubicación de los depósitos infestados con Aedes aegypti en las cuatro áreas de salud estudiadas. Municipio Playa, Ciudad de La Habana, abril de 2004 a marzo de 2005

\begin{tabular}{|c|c|c|c|c|}
\hline \multirow[b]{2}{*}{ Depósitos } & \multicolumn{4}{|c|}{ Área de salud } \\
\hline & Docente & $\begin{array}{l}28 \text { de } \\
\text { Enero }\end{array}$ & $\begin{array}{c}26 \text { de } \\
\text { Julio }\end{array}$ & $\begin{array}{l}1 .^{\circ} \mathrm{de} \\
\text { Enero }\end{array}$ \\
\hline Número de depósitos infestados & 8 & 21 & 4 & 6 \\
\hline Tanques bajos (\%) & 12,5 & 76,2 & 25,0 & 83,3 \\
\hline con larvas & 0,0 & 78,6 & 0,0 & 72,2 \\
\hline con pupas & 56,7 & 88,5 & 14,3 & 100,0 \\
\hline Bebederos (\%) & 25,0 & 9,5 & 0 & 0 \\
\hline con larvas & 0 & 10,7 & 0 & 0 \\
\hline con pupas & 6,7 & 10,8 & 0 & 0 \\
\hline Pequeños depósitos artificiales (\% ) & 50,0 & 14,3 & 75,0 & 16,7 \\
\hline con larvas & 0 & 10,7 & 0,0 & 27,8 \\
\hline con pupas & 16,7 & 0,7 & 85,7 & 0,0 \\
\hline Neumáticos (\%) & 12,5 & 0 & 0 & 0 \\
\hline con larvas & 0 & 0 & 0 & 0 \\
\hline con pupas & 20 & 0 & 0 & 0 \\
\hline Depósitos en el exterior & 87,5 & 85,7 & 75,0 & 100,0 \\
\hline Tanques tapados & 100 & 12,5 & 0 & 0 \\
\hline Tanques destapados & 0 & 25,0 & 100,0 & 80,0 \\
\hline Tanques parcialmente tapados & 0 & 62,5 & 0 & 20,0 \\
\hline Depósitos sin sombra & 0 & 9,52 & 0 & 0 \\
\hline Depósitos parcialmente sombreados & 62,5 & 57,2 & 75,0 & 66,7 \\
\hline Depósitos sombreados & 37,5 & 33,3 & 25,0 & 33,3 \\
\hline Depósitos sin materia orgánica & 0 & 9,5 & 0 & 0 \\
\hline Depósitos con poca materia orgánica & 0 & 57,2 & 25,0 & 0 \\
\hline Depósitos con mucha materia orgánica & 100,0 & 33,3 & 75,0 & 100,0 \\
\hline Presencia de vegetación & 87,5 & 81,0 & 75,0 & 100,0 \\
\hline Presencia de árboles & 71,4 & 33,3 & 33,3 & 100,0 \\
\hline
\end{tabular}

sencia de vegetación $(87,5 \%)$ y de árboles $(71,42 \%)$ alrededor de la mayoría de los depósitos encontrados. El tanque infestado se encontraba destapado.

\section{Área de salud 28 de Enero}

En esta área se revisaron en total 2617 depósitos, equivalentes a un promedio de 10,9 depósitos por casa. En todas las viviendas había agua almacenada al menos en un recipiente. Los tipos de depósito con agua más frecuentes fueron los pequeños depósitos artificiales (1 317) y los tanques bajos (439). Los menos frecuentes fueron los neumáticos (24).

En esta área se encontraron 21 depósitos positivos (cuadro 1), de ellos 7 con larvas y 14 con pupas de A. aegypti. De los depósitos positivos, 16 eran tanques bajos $(76,2 \%), 3$ pequeños depósitos artificiales $(14,3 \%)$ y 2 bebederos de animales (9,5\%). En los tanques bajos se encontró el mayor porcentaje de pupas $(88,5 \%)$, comparado con los bebederos $(10,8 \%)$ y los pequeños depósitos artificiales $(0,7 \%)$; igual comportamiento se observó con la presencia de larvas encontradas en los tanques bajos $(78,6 \%)$, bebederos $(10,7 \%)$ y pequeños depósitos artificiales $(10,7 \%)$. Se debe destacar que $85,7 \%$ de los depósitos infestados se encontraban en el exterior de las viviendas. De los 16 tanques bajos, $4(25,0 \%)$ se encontraban destapados, 10 parcialmente tapados $(62,5 \%)$ y solo $2(12,5 \%)$ estaban completamente tapados. Se encontró materia orgánica abundante en $33,3 \%$ de los depósitos y solo 9,5\% no tenían materia orgánica. La mayoría de los depósitos estaban bajo sombra parcial $(57,2 \%)$ o total $(33,3 \%)$. Si bien se encontró vegetación alrededor de la mayoría de los depósitos $(81,0 \%)$, solo la tercera parte $(33,3 \%)$ tenía árboles en su entorno. 
CUADRO 2. Resultados de la vigilancia antivectorial en las cuatro áreas de salud estudiadas. Municipio Playa, Ciudad de La Habana, abril de 2004 a marzo de 2005

\begin{tabular}{|c|c|c|c|c|c|c|c|}
\hline \multirow{2}{*}{$\begin{array}{l}\text { Área de } \\
\text { salud }\end{array}$} & \multicolumn{2}{|c|}{ Número de casas } & \multirow{2}{*}{$\begin{array}{c}\text { Índice } \\
\text { domiciliarioa }^{a}\end{array}$} & \multicolumn{2}{|c|}{ Número de depósitos } & \multirow{2}{*}{$\begin{array}{l}\text { Índice de } \\
\text { Breteau }^{\mathrm{b}}\end{array}$} & \multirow{2}{*}{$\begin{array}{l}\text { Promedio de } \\
\text { depósitos } \\
\text { por casa }\end{array}$} \\
\hline & Inspeccionadas & Infestadas & & Inspeccionados & Infestados & & \\
\hline 28 de Enero & 240 & 14 & 5,83 & 2617 & 21 & 8,75 & 10,90 \\
\hline 26 de Julio & 327 & 4 & 1,22 & 1394 & 4 & 1,22 & 4,26 \\
\hline Docente & 408 & 8 & 1,96 & 1725 & 8 & 1,96 & 4,22 \\
\hline $1 .^{\circ}$ de Enero & 265 & 5 & 1,89 & 1631 & 6 & 2,26 & 6,15 \\
\hline
\end{tabular}

a Índice domiciliario: porcentaje de casas con larvas del total de casas inspeccionadas.

b Índice de Breteau: Depósitos con larvas con relación al total de casas inspeccionadas.

\section{Área de salud 26 de Julio}

En total se revisaron 1394 depósitos (4,26 depósitos por casa en promedio) y se detectaron 6 locales sin agua almacenada. Los depósitos que más frecuentemente se encontraron con agua fueron los tanques bajos (522) y los pequeños depósitos artificiales (518); los menos frecuentes fueron los neumáticos (24).

Se hallaron solo 4 depósitos infestados (cuadro 1), de los cuales 3 (75\%) eran pequeños depósitos artificiales que se encontraban en el exterior de las viviendas y $1(25 \%)$ era un tanque bajo destapado situado en el interior. No se encontraron larvas; $85,7 \%$ de las pupas se encontraron en los pequeños depósitos artificiales. Estos contenían abundante materia orgánica, estaban parcialmente en sombra y tenían vegetación a su alrededor; uno de ellos tenía árboles cerca.

\section{Área de salud $10^{\circ}$ de Enero}

En esta área se revisaron 1631 depósitos (6,15 depósitos por casa en promedio). Solo una vivienda no tenía agua almacenada. Los tipos de depósito más frecuentes en esta área fueron los tanques bajos (653) y los pequeños depósitos artificiales (575); los menos frecuentes fueron los neumáticos, con un total de 8 .

En el área se encontraron 6 recipientes con larvas o pupas de $A$. aegypti (cuadro 1); $5(83,3 \%)$ de ellos eran tanques bajos y uno, un pequeño depósito artificial $(16,7 \%)$. Todas las pupas y la mayor cantidad de larvas $(72,2 \%)$ se encontraron en los tanques bajos. Los recipientes infestados estaban en los patios de las viviendas, contenían abundante materia orgánica y estaban a la sombra total $(33,3 \%)$ o parcial $(66,7 \%)$. En general, cuatro de los tanques estaban destapados y los demás estaban solo parcialmente tapados. Hubo presencia de vegetación y de árboles alrededor de todos los depósitos encontrados.

En resumen, en las áreas de salud Docente, 28 de Enero y $1 .^{\circ}$ de Enero, la mayor cantidad de pupas se encontró en los tanques bajos, mientras que en el área de salud 26 de Julio se encontró en los pequeños recipientes artificiales. Entre los depósitos infestados, los más frecuentes fueron los tanques bajos y los pequeños depósitos artificiales; la mayoría de ellos estaban ubicados en el exterior de las viviendas, tenían abundante materia orgánica y vegetación y árboles alrededor.

Como se muestra en el cuadro 2, en el área de salud 28 de Enero se inspeccionó el mayor número de depósitos (2 617) y se observaron los mayores valores de los índices domiciliario $(5,83)$ y de Breteau $(8,75)$. El mayor número de casas y recipientes en los que se encontraron larvas y pupas del vector del dengue estaban en las áreas de salud 28 de Enero y Docente (14 y 21, respectivamente). El mayor número promedio de depósitos por casa correspondió también al área de salud 28 de Enero, con 10,9. Al comparar el número de depósitos inspeccionados en las viviendas infestadas de las diferentes áreas estudiadas, se encontró que las áreas de salud 28 de Enero y 26 de Julio presentaron un mayor número de depósitos en las viviendas infestadas $\left(\chi^{2}=18,07 ; P=0,0004\right)$ que las dos áreas restantes, mientras que el área de salud Docente presentó un número significativamente menor de depósitos en las viviendas infestadas. En las áreas estudiadas, 4,18\% de los depósitos inspeccionados se encontraban en viviendas infestadas que tenían patio $\left(\chi^{2}=29,59 ; P=0,0001\right)$, con sombra parcial $\left(\chi^{2}=4,108 ; P=0,0001\right)$, vegetación $\left(\chi^{2}=43,59 ; P=0,0001\right)$ y árboles $\left(\chi^{2}=101,459 ; P=0,0001\right)$ y con higiene deficiente $\left(\chi^{2}=53,76 ; P=0,0001\right)$.

\section{DISCUSIÓN}

El significado de los índices domiciliario y de Breteau es aún materia de controversia y estos solo se pueden evaluar empíricamente. En Cuba, los valores de estos índices generalmente son bajos debido a la fuerte presión de control que se ejerce sobre el vector del dengue.

Los valores de los índices de Breteau y domiciliario están estrechamente correlacionados entre sí y se utilizan para calcular el riesgo de transmisión. Sin embargo, en un estudio realizado en Malasia no se encontró ninguna relación entre esos índices y el número de casos de dengue (8). Otros autores afirman que los índices larvales no reflejan adecuadamente la producción de adultos (9).

En la presente investigación se observó que una de las áreas de salud es- 
tudiadas (28 de Enero) presentó índices domiciliario y de Breteau superiores al umbral de transmisión (10), lo que demuestra la importancia de estratificar el universo de trabajo al diseñar las estrategias para el control de vectores.

En las cuatro áreas estudiadas, los depósitos en los que se encontraron larvas y pupas con mayor frecuencia fueron los tanques bajos y los pequeños depósitos artificiales, mientras que en los neumáticos estas solo se encontraron en contadas ocasiones. Otros autores también han encontrado que los principales criaderos de este vector en las áreas urbanas son los depósitos de agua para consumo humano, los pequeños depósitos artificiales, los bebederos de animales y los neumáticos (3-5, 11-14). Estos resultados coinciden también con lo encontrado en Ciudad de La Habana en el año 2005 (15) después de más de 20 años de uso de insecticidas y de trabajo masivo para erradicar los criaderos de $A$. aegypti.

La mayoría de las viviendas en las áreas de salud estudiadas tenían patios y más de $75 \%$ de todos los depósitos con larvas se encontraban en el exterior de las viviendas. Esto confirma las observaciones anteriores de otros autores $(4,6,16)$.

En todas las áreas se observó una correlación entre el número de depósitos infestados ubicados en los patios por una parte, y la presencia de vegetación y árboles, la ubicación en lugares parcial o totalmente sombreados y la higiene doméstica deficiente, por la otra. Solo en el área de salud $1 .^{\circ}$ de Enero no se encontró correlación entre el número de depósitos infestados y la presencia de árboles y de vegetación, lo cual puede deberse a que la sombra proyectada sobre los recipientes provenía de edificaciones circundantes.

La presencia de patios - cementados o no- en las viviendas se considera un factor importante que favorece la presencia de larvas de mosquitos, especialmente si en ellos se almacenan a la intemperie depósitos que se convierten en criaderos del vector cuando no existe un saneamiento adecuado. En un estudio realizado en Marilia,
Brasil, se demostró que el número promedio de criaderos de mosquitos se incrementaba a medida que disminuía el nivel económico de los moradores de la vivienda y a medida que aumentaba el tamaño del patio (17). En Taiwán, Teng y colaboradores encontraron que $63 \%$ de los criaderos de los vectores de dengue en áreas residenciales estaban en lugares abiertos en los alrededores de las viviendas (18).

En México (19), las viviendas con peores condiciones de higiene y mayor sombra presentaron mayores posibilidades de tener larvas de este mosquito. En Perú, 56\% de los criaderos de $A$. aegypti fueron lugares total o parcialmente sombreados, aunque no se encontró asociación entre el grado de infestación y la intensidad de la luz solar que llegaba a los depósitos infestados (6). A pesar de que la sombra es un factor importante, es difícil encontrar una asociación significativa, ya que depende de la localización del depósito (dentro o fuera de la vivienda) y de la utilización que se haga de él.

En el área de salud 28 de Enero se encontraron una mayor cantidad de depósitos (2 617) y un mayor número promedio de recipientes por vivienda $(10,9)$, posiblemente debido a que presentaba mayores irregularidades en el suministro de agua. No obstante, $50,3 \%$ de los depósitos encontrados eran pequeños depósitos artificiales y solo se encontraron pupas en $0,7 \%$ de ellos, posiblemente debido a que eran pequeños y la evaporación impedía el desarrollo de las larvas. Se debe destacar que los recipientes en que se encontraron pupas con mayor frecuencia fueron los tanques bajos, muy ligados a la actividad humana, ya que se empleaban para almacenar agua para uso cotidiano. Sin embargo, eso podía haberse evitado con una mayor educación y participación de la comunidad, pues $20(90,9 \%)$ de los 22 tanques en que se encontraron larvas o pupas de A. aegypti estaban total o parcialmente destapados. Los tanques tapados en que se encontraron larvas tenían las paredes deterioradas o tapas inadecuadas que permitían la entrada de las hembras del mosquito para poner sus huevos. En Perú, 97\% de los depósitos con pupas estaban destapados (6). A diferencia de las otras áreas de salud estudiadas, el área 26 de Julio mostró la mayor cantidad de pupas $(85,7 \%)$ en los pequeños depósitos artificiales (fundamentalmente en cubos), seguidos por los tanques bajos.

Otro tipo de recipiente que merece especial atención son los bebederos de animales, tanto por los resultados obtenidos en esta investigación como por lo observado en varios países $(13,14,17)$.

En las áreas estudiadas, el programa de reducción de criaderos de $A$. aegypt $i$ se debe dirigir especialmente hacia tres aspectos fundamentales: 1) la educación y participación comunitaria en el tapado correcto de los tanques bajos, así como en la aplicación del insecticida abate; 2) la correcta disposición de los recipientes y envases desechados, de manera que no estén expuestos a la lluvia; y 3) el recambio del agua en los bebederos de animales, ya que por sus características son sitios favorables para la cría del vector.

Debido a que el hábitat del mosquito vector es principalmente intra y peridomiciliario y la higiene en este entorno depende del estilo de vida de cada familia, es obvio que los sistemas de salud no pueden eliminar los criaderos del vector sin la participación activa de los ciudadanos y de la comunidad en general. Por ello, la participación de la población es el complemento necesario a los esfuerzos de los gobiernos, y para lograr el éxito es necesario informar adecuadamente a la población, vencer su apatía y crear una cultura comunitaria que promueva normas de higiene específicas.

En resumen, la vigilancia de la infestación con $A$. aegypti en las cuatro áreas de salud estudiadas permitió determinar que los depósitos artificiales, especialmente los tanques bajos y los depósitos pequeños, son las criaderos más frecuentes de $A$. aegypti, por lo que constituyen un factor de riesgo de infestación con ese vector. Los tanques destapados con materia orgánica, situados a la sombra y en exteriores son los más peligrosos en ese sentido. 


\section{REFERENCIAS}

1. Marquetti MC, Carús F, Aguilera L, Navarro A. Influencia de factores abióticos sobre la incidencia de Aedes aegypti en el Municipio 10 de Octubre, 1982-1992. Rev Cub Med Trop. 1995;47(2):131-5.

2. Orozco N, Díaz IM, Cañete A, Martínez Y. Incidencia de dengue en niños y adolescentes. Rev Cub Med Trop. 2001;53(1):16-9.

3. Tun-Lin W, Kay H, Barnes A. The premise condition index: a tool for streamlining surveys of Aedes aegypti. Am J Trop Med Hyg. 1995; 53(6):591-4.

4. Focks DA, Chadee DD. Pupal survey: an epidemiologically significant surveillance method for Aedes aegypti: an example using data from Trinidad. Am J Trop Med Hyg. 1997;56(2):159-67.

5. Focks DA. A review of entomological sampling methods and indicators for dengue vectors. Special Programme for Research and Training in Tropical Diseases. Geneva: TRD; 2003. (TRD/IDE/Den/2003).

6. Morrison AC, Astete $\mathrm{H}$, Chapilliquen $\mathrm{H}$, Ramírez Prado G, Díaz G, Getis A, et al. Evaluation of sampling methodology for rapid assessment of Aedes aegypti infestation levels in Iquitos, Peru. J Med Entomol. 2004;41(3): 502-10.

7. Armada GA, Trigo J. Manual para supervisores responsables de brigada y visitadores. Ciudad de La Habana: Ministerio de Salud Pública; 1981.
8. Sallehudin S, Zariol AP, Zulkifli A, Ahmad W. Relationship between Breteau and house indices and cases of dengue/dengue hemorrhagic fever in Kuala Lumpur, Malaysia. J Am Mosq Control Assoc. 1996:12(3):494-6.

9. Service MW. Importance of ecology in Aedes aegypti control. Southeast Asian J Trop Med Public Health. 1992:23(4):681-90.

10. Fernández I. Biología y control de Aedes aegypti. Manual de operaciones. Monterrey (México): Universidad Autónoma de Nuevo León; 1999.

11. Pattamaporn K, Strickman D. Distribution of container-inhabiting Aedes larvae (Diptera: Culicidae) at a dengue focus in Thailand. J Med Entomol. 1993;30(3):600-6.

12. Vezzani D, Schweigmann N. Suitability of containers from different sources as breeding sites of Aedes aegypti (L) in a cementery of Buenos Aires City, Argentina. Mem Inst Oswaldo Cruz. 2002;97(6):789-92.

13. Strickman D, Pattamaporn K. Dengue and its vectors in Thailand: collected transmission risk from total pupal counts of Aedes aegypti and association of wing-length measurements with aspects of the larval habitat. Am J Trop Med Hyg. 2003;68(2):209-17.

14. Hayes J, García E, Flores R, Suárez G, Rodríguez T, Coto R, et al. Risk factors for infection during a severe dengue outbreak in El Salvador in 2000. Am J Trop Med Hyg. 2003;69(6); 629-33.
15. Marquetti MC, Suárez S, Bisset J, Leyva M. Reporte de habitats utilizados por Aedes aegypti en Ciudad de La Habana, Cuba. Rev Cub Med Trop. 2005;57(2):159-61.

16. Nathan MB, Giglioli ME. Erradicación de Aedes aegypti en Caiman Brac y Pequeño Caimán, Antillas Británicas, con abate (temephos) en 1970-1972. Bol Oficina Sanit Panam. 1973;92(1):18-32.

17. Mazine $C A B$, Macorís MLG, Andrighetti MJM, Yasumaro S, Silva ME, Nelson MJ, et al. Disposable containers as larval habitats for Aedes aegypti in a city with regular refuse collection: a study in Marilia, São Paulo state, Brazil. Acta Tropica. 1996;62(57):1-13.

18. Teng HJ, Wu YL, Ting HS. Mosquito fauna in water-holding containers with emphasis on dengue vectors (Diptera: Culicidae) in Chungho, Taipei Country, Taiwan. J Med Entomol. 1999;36(4):468-72.

19. Espinoza GF, Hernández SM, Coll CR. Factores que modifican los índices larvarios de Aedes aegypti en Colima México. Rev Panam Salud Publica. 2001;10(1):6-12.

Manuscrito recibido el 26 de abril de 2006. Aceptado para publicación, tras revisión, el 15 de mayo de 2006.
ABSTRACT

Ecological factors linked to the presence of Aedes aegypti larvae in highly infested areas of Playa, a municipality belonging to Ciudad de La Habana, Cuba

Key words
Objectives. To identify local ecological factors that might have had an impact on the higher infestation rates by the vector mosquito Aedes aegypti seen in four urban health districts of Ciudad de La Habana, Cuba, and to determine the type of containers with the highest number of larvae.

Methods. This is a descriptive study that was carried out in four health areas (Docente, 28 de Enero, 26 de Julio, and $1 .^{\circ}$ de Enero) within Playa, a municipality belonging to Ciudad de La Habana, Cuba. These areas were chosen because of the persistent presence of the dengue vector throughout 2003. All buildings on the selected blocks were inspected between April 2004 and March 2005, and note was taken of existing water deposits (type, number, and location) and of surrounding ecological factors (existence of a patio and shade, the way tanks were covered, the presence of organic matter inside them, and the overall cleanliness of the dwelling). Using filtration, the mosquito larvae and pupae in each deposit were counted. Pupae were typified as to their taxonomic species under a stereoscopic microscope.

Results. The types of deposits where larvae and pupae of A. aegypti were most often found in all areas were low tanks and small artificial containers. In three of the study areas the greatest numbers of pupae were found in low tanks $(88.6 \%, 100 \%$, and $56.6 \%), 90.9 \%$ of which were uncovered or only partially covered. On the other hand, in the fourth study area small artificial deposits were the most commonly infested $(85.7 \%)$. A correlation was noted between the number of deposits in infested houses and the presence of a patio $\left(\chi^{2}=29.59 ; P=0.0001\right)$, partial shade $\left(\chi^{2}=4.108 ; P=\right.$ $0.0001)$, shrubbery $\left(\chi^{2}=43.59 ; P=0.0001\right)$ and trees $\left(\chi^{2}=101.459 ; P=0.0001\right)$, as well as poor hygiene $\left(\chi^{2}=53.76 ; P=0.0001\right)$.

Conclusions. Artificial deposits, especially low tanks and small containers, are the most frequent breeding sites for $A$. aegypti and are therefore risk factors for infestation with this vector species. Uncovered tanks containing organic matter and located in the shade on the outside of homes were found to present the highest risk in this respect.

Aedes, mosquito control, epidemiologic surveillance, Cuba. 